\title{
Dysregulation of oncogenic factors by GFI1B p32: investigation of a novel GFI1B germline mutation
}

\author{
Michela Faleschini, ${ }^{1}$ Nicole Papa, ${ }^{1}$ Marie-Christine Morel-Kopp, ${ }^{2}$ Caterina \\ Marconi, ${ }^{3}$ Tania Giangregorio, ${ }^{1}$ Federica Melazzini, ${ }^{4}$ Valeria Bozzi, ${ }^{4}$ Marco \\ Seri, ${ }^{3}$ Patrizia Noris, ${ }^{4}$ Alessandro Pecci, ${ }^{4}$ Anna Savoia ${ }^{1,5}$ and Roberta Bottega ${ }^{1}$ \\ ${ }^{1}$ Institute for Maternal and Child Health - IRCCS Burlo Garofolo, Trieste, Italy; \\ ${ }^{2}$ Department of Hematology and Transfusion Medicine, Royal North Shore Hospital \\ and Northern Blood Research Center, Kolling Institute, University of Sydney, Sydney, \\ New South Wales, Australia; ${ }^{3}$ Department of Medical and Surgical Sciences, University \\ of Bologna, Bologna, Italy; ${ }^{4}$ Biotechnology Research Laboratories, IRCCS Policlinico \\ San Matteo Foundation, Pavia, Italy and ${ }^{5}$ Department of Medical Sciences, University \\ of Trieste, Trieste, Italy
}

Haematologica 2022

Volume 107(1):260-267

\section{ABSTRACT}

C FI1B is a transcription factor essential for the regulation of erythropoiesis and megakaryopoiesis, and pathogenic variants have been associated with thrombocytopenia and bleeding. Analysing thrombocytopenic families by whole exome sequencing, we identified a novel $G F I 1 B$ variant (c. $648+5 G>A)$, which causes exon 9 skipping and overexpression of a shorter p32 isoform. We report the clinical data of our patients and critically review the phenotype observed in individuals with different GFI1B variants leading to the same effect on the p32 expression. Since p32 is increased in acute and chronic leukemia cells, we tested the expression level of genes playing a role in various type of cancers, including hematological tumors and found that they are significantly dysregulated, suggesting a potential role for $G F I 1 B$ in carcinogenesis regulation. Increasing the detection of individuals with $G F I 1 B$ variants will allow us to better characterize this rare disease and determine whether it is associated with an increased risk of developing malignancies.

\section{Correspondence:}

ANNA SAVOIA

anna.savoia@burlo.trieste.it

Received: July 31, 2020.

Accepted: December 21, 2020.

Pre-published: January 21, 2021.

https://doi.org/10.3324/haematol.2020.267328

(C)2022 Ferrata Storti Foundation

Material published in Haematologica is covered by copyright. All rights are reserved to the Ferrata Storti Foundation. Use of published material is allowed under the following terms and conditions:

https://creativecommons.org/licenses/by-nc/4.0/legalcode. Copies of published material are allowed for personal or internal use. Sharing published material for non-commercial purposes is subject to the following conditions:

https://creativecommons.org/licenses/by-nc/4.0/legalcode, sect. 3. Reproducing and sharing published material for commercial purposes is not allowed without permission in writing from the publisher.

\section{Introduction}

Inherited thrombocytopenias represent a group of heterogeneous rare disorders characterized by a reduced platelet count that may associate with other defects. ${ }^{1}$ Mutations in at least 40 different genes are responsible for these disorders, including a recently discovered autosomal dominant condition reported as GFI $B$-associated bleeding disorder or platelet-type bleeding disorder 17 (OMIM 187900), which is caused by pathogenic variants of the GFI1B (growth factor-independent 1B) gene..$^{2-4}$

GFI1B is a transcription repressor of the GFI zinc-finger family, consisting of three domains: a highly conserved N-terminal repressor domain called Snail/Gfil, which recruits chromatin regulatory proteins; an intermediary domain of unknown function; and a C-terminal cluster of six zinc-finger domains (ZNF), of which ZNF 3, 4, and 5 bind to DNA, while ZNF 1,2, and 6 have yet to be characterized. ${ }^{5,6}$ GFI1B plays a key role in hematopoiesis through different isoforms. Indeed, in addition to generating a long isoform consisting of 330 amino acids (p37), GFI1B mRNA undergoes an alternative splicing process producing a short isoform of 284 residues (p32) characterized by the skipping of exon 9 (NG_034227) and consequent removal of ZNF 1 and 2. The long p37 form is reported to have a pivotal role in megakaryopoiesis and platelet production, whereas the short p32 form is essential for erythroid lineage.'

GFI1B regulates the differentiation of the hematopoietic stem cells through the repression of different promoters, including its self-regulatory regions via an autoregulatory feedback mechanism. ${ }^{7-9}$ Among other targets is the hematopoietic stem cell marker CD34, whose downregulation during hematopoiesis is not quenched when GFI1B is mutated. Therefore, one feature of the GFI1B-associated bleeding disorder is the aberrant expression of CD34 on patients' platelets, which is due to the dysreg- 
ulation of the respective gene at the transcriptional level. ${ }^{10}$

Since its first description, ${ }^{2}$ individuals with GFIB $B$ mutations have been examined in order to characterize this novel rare platelet disorder. To our knowledge, these studies have identified at least 15 different mutations in approximately 20 unrelated families, allowing better characterization of the disease, whose features are increased bleeding tendency, thrombocytopenia with enlarged platelets and a reduced $\alpha$-granule content with abnormal distribution. ${ }^{2,3,10-17}$

We report a novel heterozygous GFI1B variant (c.648+5G>A) in a family with mild thrombocytopenia and no other significant features. The substitution causes skipping of exon 9 and consequent overexpression of the short p32 isoform and dysregulation of GFI1B target genes, including CD34 and other genes that are involved in neoplastic transformation, suggesting a potential role for GFI1B in carcinogenesis regulation.

\section{Methods}

\section{Family study and clinical features}

The propositus (Figure 1), a 65 -year-old female with low platelet number, and her family members were studied to determine the molecular basis of thrombocytopenia. Their clinical features, as well as the methods used for blood cell analyses, ${ }^{1,19}$ are described in more detail in the Online Supplementary Appendix. The Institutional Review Board of the IRCCS "Policlinico San Matteo
Foundation" of Pavia approved the study. All subjects provided written informed consent for the study, which was conducted in accordance with the Declaration of Helsinki.

\section{Mutation screening and reverse transcriptase polymerase chain reaction analysis}

Mutational screening was performed by whole exome sequencing (WES) in the proband (II-2), as previously reported. ${ }^{20}$ Variants were confirmed by Sanger sequencing in her daughters (III-1 and III-2), sisters (II-3 and II-4) and brother (II5) (Table 1). Total RNA was extracted from peripheral blood cells of patients II-2 and III-1, as well as two healthy controls and cDNA amplified as previously reported, ${ }^{21}$ using primers enlisted in the Online SupplementaryTable S1.

\section{Bioinformatic analysis}

The effect of the splice-site mutation was predicted by in silico analyses using two dedicated bioinformatic tools: splice site prediction by Neural Network (NNSplice; http://www.fruitfly.org/seq_tools/splice.html) and Human Splicing Finder Version 2.4.1 (http://www.umd.be/HSF/).

\section{Expression vectors and dual-Iuciferase reporter assay}

Wild-type and mutant (c.511_648del due to skipping of exon 9) $G F I 1 B$ cDNA were cloned into the tagged (myc) expression vector pcDNA3.1 (Invitrogen, Carlsbad, CA, USA). The GFI1B, GFI, $M E I S 1$, and CD34 promoters were cloned into a reporter firefly luciferase vector (pGL4/luc2, Promega). Plasmids were transiently
A

e9

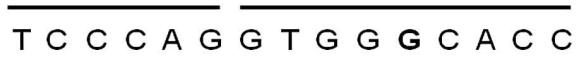
$T \subset C \subset A G G T G G A \subset A \subset C$ wt

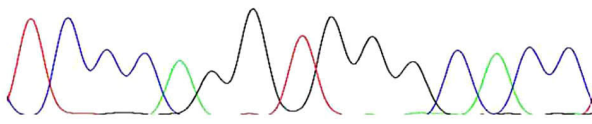

II-2

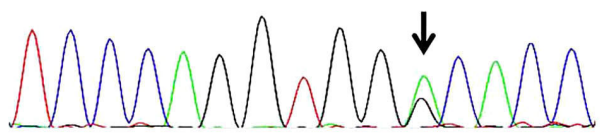

B

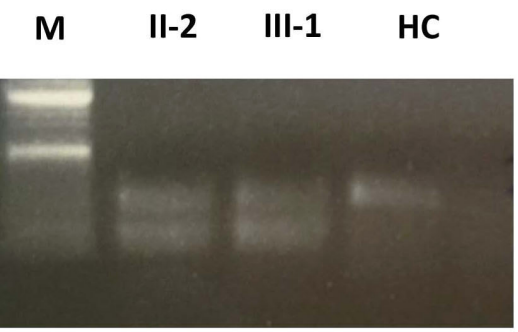

bp

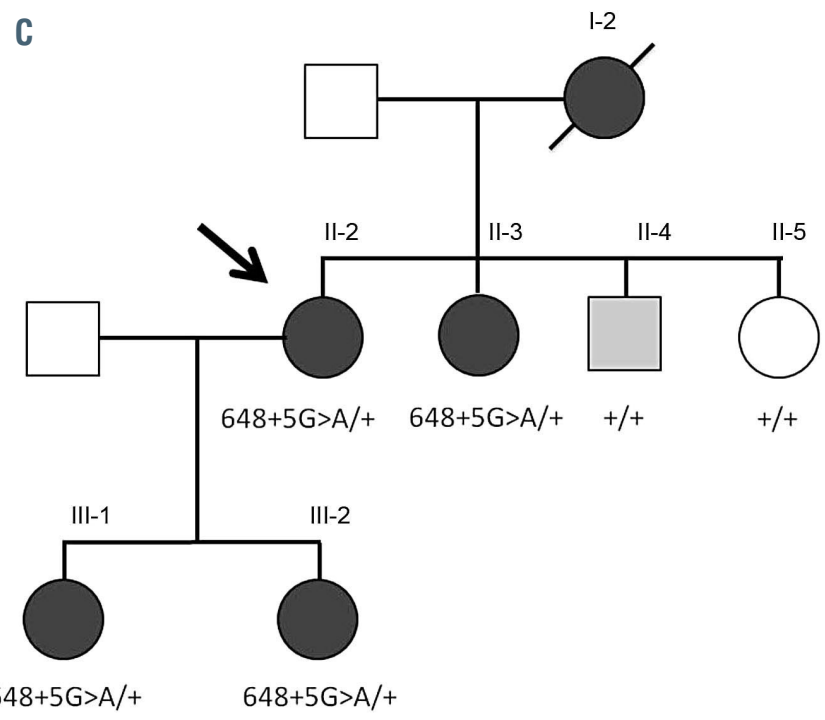

Figure 1 Genetic analyses of the family. (A) Sange sequencing showing exon 9 (e9) and intron 9 (i9) boundary of GFI1B gene. The heterozygous c. $648+5 \mathrm{G}>\mathrm{A}$ substitution is indicated by an arrow. Nucleotide A of the ATG translation initiation start site of the GFI1B cDNA in GenBank sequence NM_004188.6 is indicated as nucleotide +1 . (B) Real-time polymerase chain reaction showing different transcription pattern between patients (II-2; III-1) and healthy control (HC). The expected product of 246 basepairs (bp) was detected in the $\mathrm{HC}$ sample; an additional band of $108 \mathrm{bp}$ corresponding to skipping of exon 9 was found in the affected individuals. $\mathrm{M}$ : molecular weight. (C) Family pedigree showing an autosomal dominant pattern of inheritance. Arrow indicates the proband whereas black filled symbols represent affected individuals carrying the mutation. The grey filled symbol indicates a thrombocytopenic brother without the mutation (platelet count in this patient may be influenced by a liver cirrhosis). wt: wild-type. 
co-transfected in Meg01, as previously described. ${ }^{2}$ The luciferase activity was detected using the Dual Luciferase reporter assay system kit (Promega), according to the manufacturer's instructions. Details of the experiments are included in the Online Supplementary Appendix.

\section{Quantitative real-time polymerase chain reaction}

Quantitative real-time polymerase chain reaction (qRT-PCR) was performed to evaluate the absolute expression of GFI1B isoforms and relative expression of $G F I 1 B$ major target, using specific primers (Online Supplementary Table S2). FastStart Universal SYBR Green Master Mix (Roche) and ABI PRISM 7900 detection (Applied Biosystem, Foster City, CA, USA) were used. All experiments were performed three times in triplicate, as previously reported. ${ }^{22}$

\section{Western blot analyses}

HEK293T cells were transfected with myc-tagged wild-type and mutant GFI1B cDNA using calcium phosphate method and maintained in DMEM medium (Euroclone, Pero, Italy) with 10\% fetal bovine serum. Protein fractionated cell extracts were analyzed by western blot using primary antibodies anti-myc (9E10; sc-40; Santa Cruz Biotechnology, Dallas, TX, USA), anti-HSP90 (sc-7947; Santa Cruz Biotechnology), anti-ORC2 (ab68348; Abcam, Cambridge, UK); as previously described. ${ }^{23}$

\section{Immunofluorescence assay}

GFIB transfected in HeLa cells was detected using 9E10 antibody against c-myc (Santa Cruz Biotechnology). Peripheral blood smears were double-labeled with antibodies against CD41 (Santa Cruz Biotechnology) and CD34 (Miltenyi Biotec, Bergisch Gladbach, Germany). General staining and image acquisition have previously been described. ${ }^{24,25}$ Detailed methods for both immunofluorescence analyses are included in the Online Supplementary Appendix.

\section{Results}

\section{Identification of the novel c.648+5G>A mutation in GFI1B}

WES of proband II-2 revealed a single nucleotide heterozygous substitution (c.648+5 G>A) in the GFI1B gene, affecting the splice donor site of intron 9 (Figure 1A). Sanger sequencing confirmed the variant in the proband (II-2), her sister (II-3) and her daughters (III-1 and III-2) but not in her healthy sister (II-5) (Figure 1B). The proband's brother (II-4) was homozygous for the wild-type allele and the thrombocytopenia was likely due to liver cirrhosis. The c.648+5G >A substitution is reported in GnomAD with a microcytic anemia factor (MAF) value of 0.000008025 and identified in two European (non-Finnish) individuals in heterozygous status.

Based on NNSplice software, the c. $648+5 \mathrm{G}>\mathrm{A}$ variant is predicted to drop the score of the splice donor site from 0.89 to 0.24 , suggesting potential alternative splicing processes. Therefore, RT-PCR analysis was performed using RNA extracted from affected individuals' peripheral blood cells (II-2 and III-1) (Figure 1C). In contrast with the healthy control showing the expected product of 246 basepairs (bp), the patients' samples amplified an additional band of $108 \mathrm{bp}$. Sequencing analysis revealed that the lower band corresponded to a transcript characterized by skipping of exon 9, resulting in an in-frame mRNA deletion of $138 \mathrm{bp}$ (p.Val171_Gln216del). The alternative splicing is expected to produce a shorter protein of $32 \mathrm{kDa}$, lacking zinc fingers 1 and 2, corresponding to what was previously described as p32. ${ }^{7.26}$

\section{Blood cell studies in family members}

The major blood parameters of the family members are reported in Table 1. The propositus had moderate thrombocytopenia while the other individuals carrying the heterozygous c.648+5G>A GFI1B mutation had a platelet count only slightly lower or higher than the lower limit of the normal range. Platelet size calculated as mean platelet volume (MPV) by the automated counter was normal in all subjects. The study of myeloproliferative disorders (MPD) by image analysis of blood smears revealed slightly increased values, indicating mild platelet macrocytosis. The remaining parameters, including hemoglobin concentration, mean red cell volume (MCV) and red blood cell count were within the normal ranges in all investigated subjects. Examination of peripheral blood smears revealed a mild reduction in platelet $\alpha$-granules and red blood cell anisocytosis in all affected family members. Flow cytometry revealed normal expression of the platelet surface GP complexes Ia-IIa, IIb-IIla, and Ib-IX-V in individuals II-2 and III1 (data not shown). Platelet aggregation after stimulation with collagen (4 and $20 \mu \mathrm{g} / \mathrm{mL}), \mathrm{ADP}(5$ and $20 \mu \mathrm{M}$,) epinephrine $(10 \mu \mathrm{M})$, arachidonic acid $(1 \mu \mathrm{M})$, TRAP $(25 \mu \mathrm{M})$, and ristocetin $(1.5 \mathrm{mg} / \mathrm{mL})$ was normal in individuals II-2

Table 1. Clinical and laboratory parameters in GFI1B mutated and non-mutated family members

\begin{tabular}{|c|c|c|c|c|c|c|c|c|c|c|}
\hline $\begin{array}{l}\text { Subject } \\
\text { (Sex) }\end{array}$ & $\begin{array}{c}\text { GFI1B } \\
\text { mutational } \\
\text { status }\end{array}$ & $\begin{array}{l}\text { Platelet } \\
\text { count } \\
\left(x 10^{9} / L\right)\end{array}$ & MPV (fiL) & MPD ( $(\mu \mathrm{m})$ & Hb (g/dL) & MCV (iㄴ) & $\begin{array}{c}\text { RBC } \\
\left(\times 10^{6} / \mu L\right)\end{array}$ & RCDW (\%) & $\begin{array}{c}\text { Leukocyte } \\
\text { count } \\
\left(x 10^{9} / L\right)\end{array}$ & $\begin{array}{l}\text { WHO } \\
\text { bleeding } \\
\text { score }^{\S}\end{array}$ \\
\hline II-2 (F) & $648+5 \mathrm{G}>\mathrm{A} /+$ & 50 & 10.7 & 3.32 & 13.6 & 85 & 4.89 & 14 & 6.23 & 3 \\
\hline II-3 (F) & $648+5 \mathrm{G}>\mathrm{A} /+$ & 149 & 9.2 & 2.95 & 13.4 & 90.6 & 4.40 & 13.7 & 4.01 & 3 \\
\hline III-1 (F) & $648+5 \mathrm{G}>\mathrm{A} /+$ & 124 & 12.6 & 2.96 & 12 & 82.3 & 4.10 & 13.4 & 7.62 & 0 \\
\hline III-2 (F) & $648+5 \mathrm{G}>\mathrm{A} /+$ & 153 & 11.7 & 3.57 & 13.9 & 88.3 & 4.80 & 15 & 9.1 & 0 \\
\hline II-4 (M) & $+/+$ & $94 *$ & 10.8 & 3.09 & 15.6 & 98 & 4.92 & 13.9 & 5.7 & 0 \\
\hline II-5 (F) & $+/+$ & 215 & 10 & 3.26 & 14.6 & 95.1 & 4.58 & 14 & 5.42 & 0 \\
\hline Normal values & & $150-450$ & $8-13^{* *}$ & $2.4-2.7 \dagger$ & $\begin{array}{l}11.7-15.5(\mathrm{~F}) \\
13.2-17.3(\mathrm{M})\end{array}$ & $82-98$ & $\begin{array}{c}3.8-5.2(\mathrm{~F}) \\
4.4-5.7(\mathrm{M})\end{array}$ & $11.6-16$ & $4-10$ & 0 \\
\hline
\end{tabular}

${ }^{s}$ F: female; M: male; * in this patient thrombocytopenia was likely due to liver cirrhosis; ** MPV range given by the automated cell counter; $\dagger$ MPD obtained in 50 healthy volunteers $(95 \%$ confidence interval) 18; §World Health Organization (WHO) bleeding scale: 0 - no bleeding tendency; 1 - cutaneous bleeding only (including minimal mucosal bleeding); 2 - mild blood loss (any mucosal bleeding not fulfilling the criteria for grade 1 or 3 ); 3 - gross blood loss, requiring transfusion; 4 - debilitating blood loss (including retinal or cerebral associated with fatality). See patients and methods for details about bleeding symptoms. MPV: mean platelet volume; MDP: myeloproliferative disorders; Hb: hemoglobin; RBC: red blood cell. 
and III- 1 carrying the c. $648+5 \mathrm{G}>\mathrm{A}$ variant, and similar to II4 who is wild-type (data not shown).

\section{Expression level of alternative spliced products}

The patient's cDNA was used to clone the wild-type (330 amino acids [aa]; p37) and the exon 9 skipped (284 aa; p32) forms of GFIAB tagged to myc into an expression vector. Accordingly to what was observed during the RT-
PCR on patients RNA (Figure 1C), colony screening using primers on exons 8 and 10 (Online Supplementary Table S1) detected the expected products of $246 \mathrm{bp}$ and of $108 \mathrm{bp}$, as well as two additional bands of $312 \mathrm{bp}$ and $174 \mathrm{bp}$ (Figure 2A). Sanger sequencing showed that the additional products retained the last $66 \mathrm{bp}$ (gggatccogecgggtccagtcctgagcctgcacctgacccccogggectcatttcctccggcag) of intron 9 in both p37 and p32 forms due to the recogni-

A

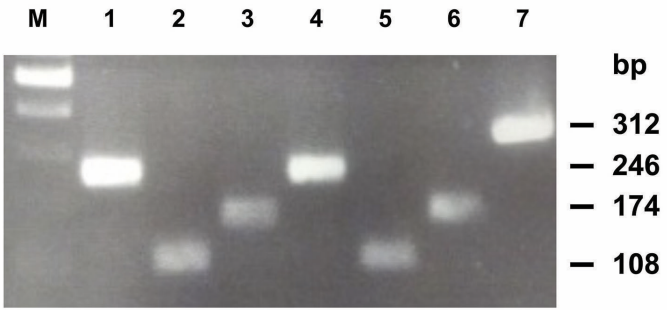

B
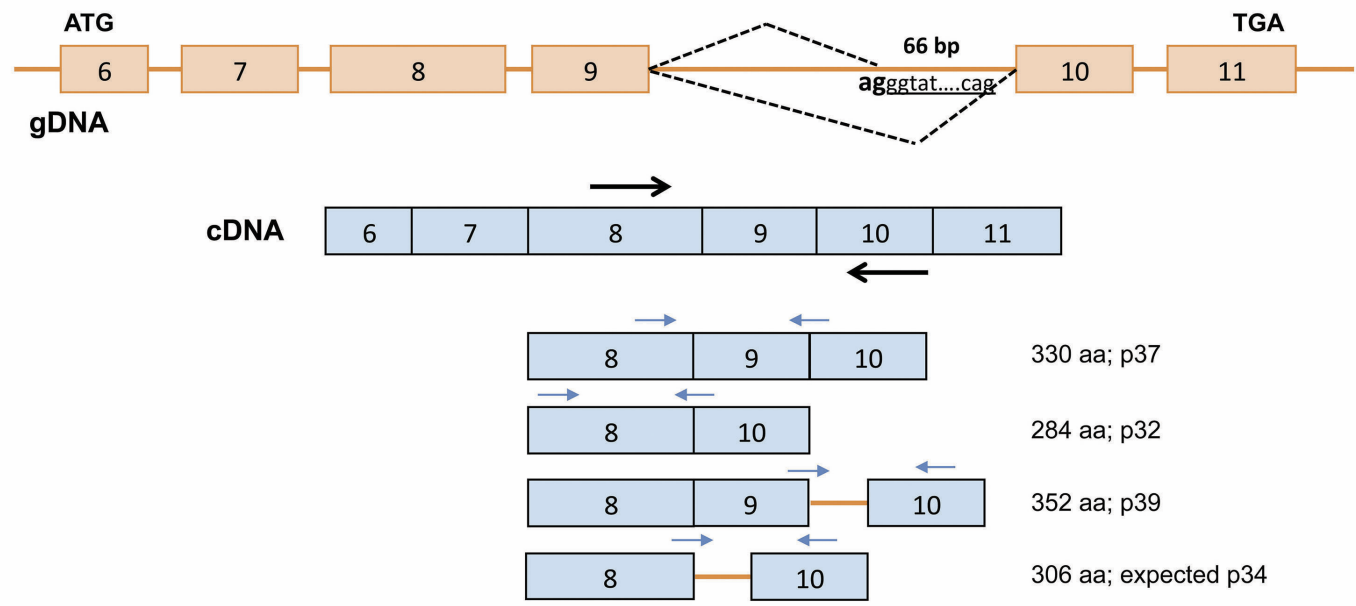

330 aa; p37

284 aa; p32

352 aa; p39

306 aa; expected p34

C

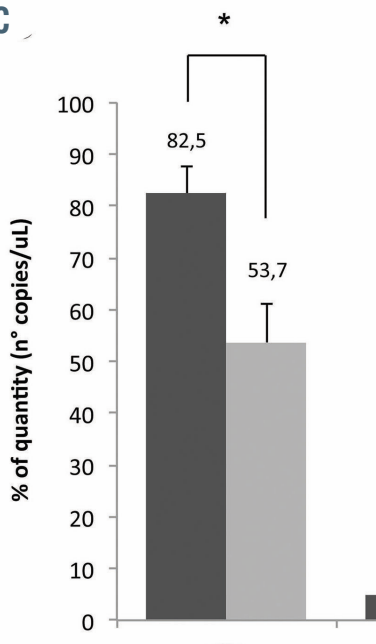

p37

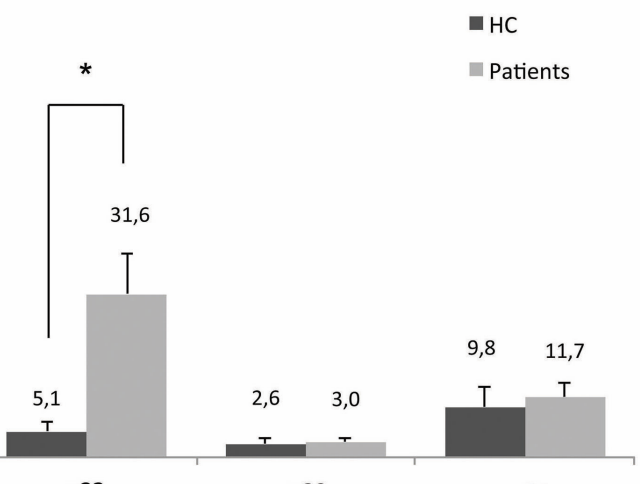

Figure 2. GFI1B splicing isoforms. (A) Polymerase chain reaction (PCR) products obtained after amplification of a portion of GFI1B from different plasmids containing subcloned GFI1B cDNA (individual II-2) using primer aligning on exons 8 and 10. Fragments corresponding to the wild-type (246 basepairs [bp]) and skipped exon 9 (108 bp) forms of GFI1B are shown in lanes 1 and 4, and 2 and 5, respectively. Additional fragments of 312 bp (lane 7 ) and 174 bp (lanes 3 and 6 ) corresponding to $\mathrm{p} 39$ and p34, respectively, were also detected. M: molecular weight. (B) Schematic structure of the genomic GFI1B gene (gDNA) which includes the coding exons (exons 6-11; NM_004188.6). On the bottom, the representation of the full cDNA (p37) and the different isoforms identified. p32 is characterized by skipping of exon 9. p39 and p34 results in p37 and p32, respectively, which also retain the last 66 bp of intron 9 (of which the first and the last are listed in detail) due to recognition of a cryptic acceptor splice site "ag" (in bold). Arrows represent primers used for the amplification. (C) Differential expression level of the four GFI1B isoforms between patients (II-2 and III-1) and healthy controls (HC) perfomed by quantitative PCR using specific primers. Overall the wild-type isoform (p37) is the most represented in controls while p32 is significantly increased in patients $(* P<0.05)$. Error bars represent the standard deviation of three independent experiments. Statistical analysis was performed using $t$-test. GDNA: genomic DNA; cDNA: coding DNA; aa: amino acids. 
tion of a cryptic acceptor splicing site (ggagtgtcctgttccgcaggggat) with a score of 0.83 predicted by NNSpice tool (Figure $2 \mathrm{~B}$ ). Therefore, the two additional products would correspond to GFI1B molecules of an expected molecular weight of $39 \mathrm{kDa}(\mathrm{p} 39)$ and $34 \mathrm{kDa}(\mathrm{p} 34)$, respectively for an in-frame insertion of 22 aa (p.216_217insGIPAGSSPEPAPDPPGPHFLRQ).

We investigated the expression level of the four forms of GFI1B (Online Supplementary Table S2). In control samples $(\mathrm{n}=2)$, forms p37 and p32 represented the most expressed products, being approximately $83 \%$ and $10 \%$ of the total GFI1B cDNA, respectively; the other two forms, p34 and p39, were overlooked (Figure 2C). In patients, the expression level of p37 and p32 was significantly different than in controls, corresponding to $54 \%$ and $32 \%$ of the total cDNA, respectively. Of note, p32 was relatively less expressed than p37, suggesting that c. $648+5 \mathrm{G}>\mathrm{A}$ leads to partial skipping of exon 9 or partial degradation of the $\mathrm{mRNA}$. Finally, no significant difference in the expression of p39 and p34 was observed between patient and control samples.

\section{Pathogenic role of the $\mathrm{c.648+5G>A}$ mutation leading to p32}

In order to investigate the effect of c. $648+5 \mathrm{G}>\mathrm{A}$ on $\mathrm{GFI} 1 \mathrm{~B}$ cellular localization, we performed immunofluorescence and western blot analysis in cells transiently transfected with the wild-type or mutant cDNA. Like p37, the p32 isoform enters the HeLa cell nucleus (Figure $3 \mathrm{~A}$ ) and both forms are similarly distributed in the cytoplasmic and nuclear fractions of Hek293 cells (Figure 3B), suggesting that zinc fingers 1 and 2 are dispensable for migration of p32 into the nucleus.

Moreover, we determined the effect on the transcriptional activity, using the luciferase gene under the control of three known GFI1B target gene promoters (MEIS, GFI, and GFI1B itself). The luciferase activity was significantly reduced when the Meg-01 cells were co-transfected with myc-tagged p37, confirming the role of GFI1B as a transcription repressor of those three targets. ${ }^{27} \mathrm{On}$ the contrary, the expression of p32 not only abolished the repression but also increased the transcriptional activity of those three promoters, suggesting that the functional defect is likely due to
A

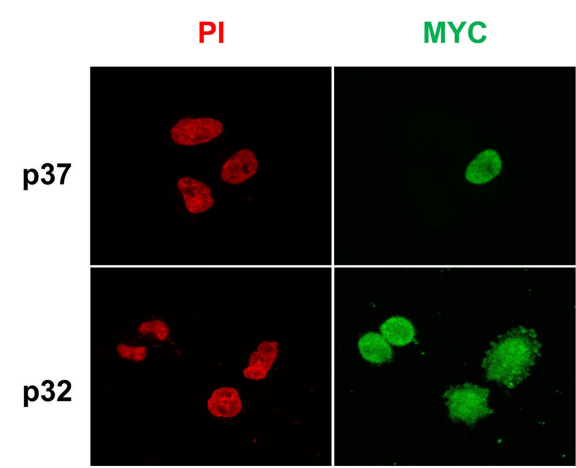

MERGE

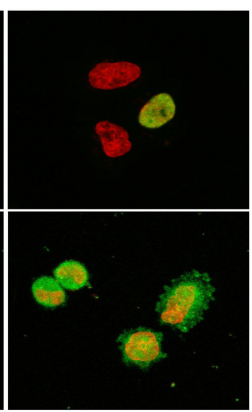

B

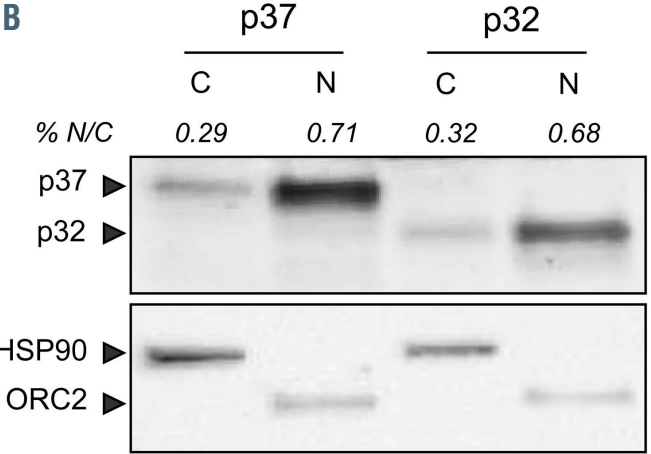

C

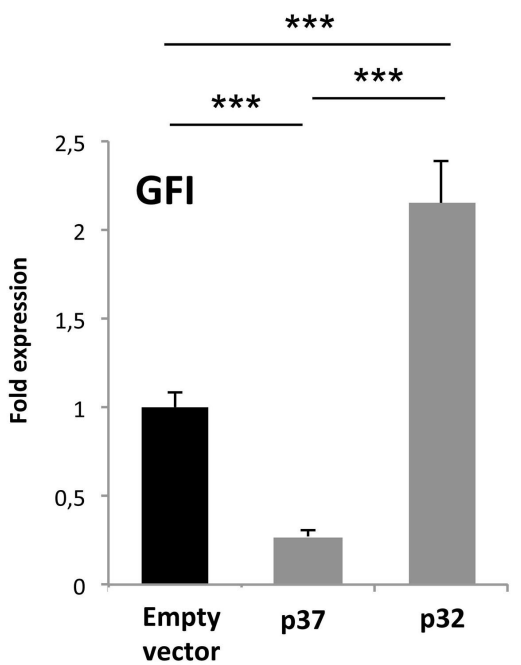

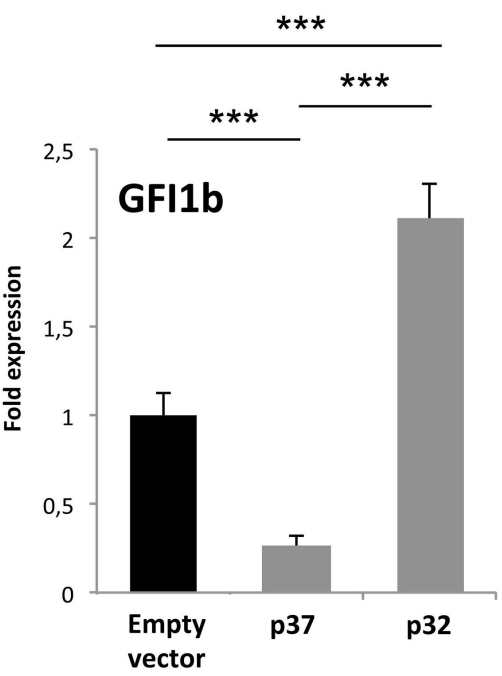
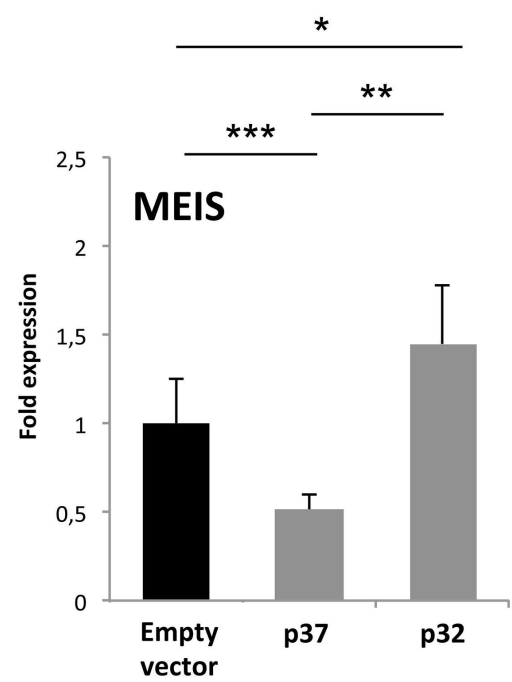

Figure 3. Pathogenic role of GFI1B p32. (A) Immunofluorescence (IF) and (B) western blot (WB) of nuclear (N) and cytoplasmatic (C) fractionated cellular lysates from Hek293 transiently transfected with wild-type (WT) (p37) and mutated (p32) GFI1B expression vector. Results demonstrate that both isoforms are distributed in the nucleus as well as in the cytoplasm with the same proportions. In IF, propidium iodide (PI) was used to detect nuclei. HSP90 and ORC2 antibodies were used in WB as loading marker for the cytoplasmic and nuclear fraction, respectively. (C) Transcriptional activity of p37 (WT) and the variant p32 GFI1B isoforms measured on GFI, GFIIB and MEIS promoters in Meg01 cells. Results are represented by ratio of the promoter signal (Firefly) and the control promoter (Renilla) and expressed as fold change in luciferase activity. p37 shows the expected repressive activity on targets compared to the empty vector while p32 is an activator (1- to >1.5-fold) in comparison with the p37 for all the three targets $(* * * p<0.001)$. Error bars represent the standard deviation of three independent experiments. Statistical analysis was performed using $t$-test. 
a dominant negative effect of the exogenous p32 on the p37 endogenous transcription factor in Meg-01 cells (Figure 3C). Of note, similar data were obtained using non-tagged p37 and p32 forms generated to test the effect of a different mutation (c.648+1_648+8delGTGGGCAC) of GFI1B..$^{10}$

\section{Transcriptional regulation of the CD34 promoter reflects} the aberrant expression of CD34 in patients' platelets

Previous investigations reported that GFI1B-related thrombocytopenia is associated with aberrant expression of the stem cell antigen CD34 in platelets and megakaryocytes, which could be caused by the loss of CD34 downregulation during megakaryocytopoiesis due to defective GFI1B function. ${ }^{10}$ We therefore investigated the transcriptional effect of p32 on the CD34 promoter. Similarly to what observed on $M E I S, G F I$, and GFI1B promoters, p32 does not repress the CD34 promoter activity $(P<0.001)$ (Figure 4A). Consistent with our in vitro findings, immunofluorescence analysis of peripheral blood smears confirmed the aberrant expression of the CD34 antigen in the proband's platelets (II-2) (Figure 4B).

\section{p32 dysregulates the transcription of genes involved in oncogenic pathways}

Since myeloid and lymphoid leukemia cells express higher level of p32 than control cells, ${ }^{26}$ we hypothesized that p32 could unbalance the transcription of genes involved in malignant transformation. For this reason, we investigated the expression level of other GFI1B targets, including protooncogenes GFI1 and MYC, antiapoptotic factors TGFBR3 and BCL2L1, and hematopoietic master regulators, the GATA family members, whose dysregulation is known to occur in oncogenesis. qPCR performed on patients' peripheral blood RNA showed that except for GATA2, all the other genes tested (GFI1, BCL2L1, TGFBR3, MYC, GATA1 and GATA3) were significantly overexpressed in patients II2 and III-1 compared to controls with a fold-change ranging from 1.5 to $>3$ (Figure 5), suggesting that patients carrying the c.648+5G $>$ A mutation could have an increased risk for malignancies. In order to confirm the role of p37 and p32 in regulating the oncogenic factors, $\mathrm{QPCR}$ was also carried out in HEK cells overexpressing the two GFI1B isoforms. As in patients' peripheral blood cells, the overexpression of p32 is associated with an increased expression level of the oncogenes, indicating that p32 up-regulates their transcription (Online Supplementary Figure S1). The only exception is represented by GATA 3, whose expression was significantly decreased in p32-overexpressing cells instead of being increased as in patients' samples, suggesting that p32 downregulates this gene at least in the HEK cellular model.

\section{Discussion}

GFI1B is a transcriptional repressor that plays a fundamental role in megakaryocyte development and erythropoiesis $^{9}$ and pathogenic variants are responsible for the GFIB $B$-associated bleeding disorder. The novel heterozygous $G F I 1 B$ splicing variant (c. $648+5 \mathrm{G}>\mathrm{A}$ ) identified in our family causes alternative splicing leading to unbalanced expression level of the GFI1B isoforms. Specifically, we detected four alternative splicing events, leading to the three known isoforms, p37, p32 and p39, as well as the novel p34 isoform. The p37 and p32 isoforms are expressed at different level in the individuals carrying the c. $648+5 \mathrm{G}>\mathrm{A}$ substitution, with the p32/p37 ratio ten times higher in affected than healthy individuals, indicating that this unbalance, due to the presence of the c. $648+5 \mathrm{G}>\mathrm{A}$ mutation,
A

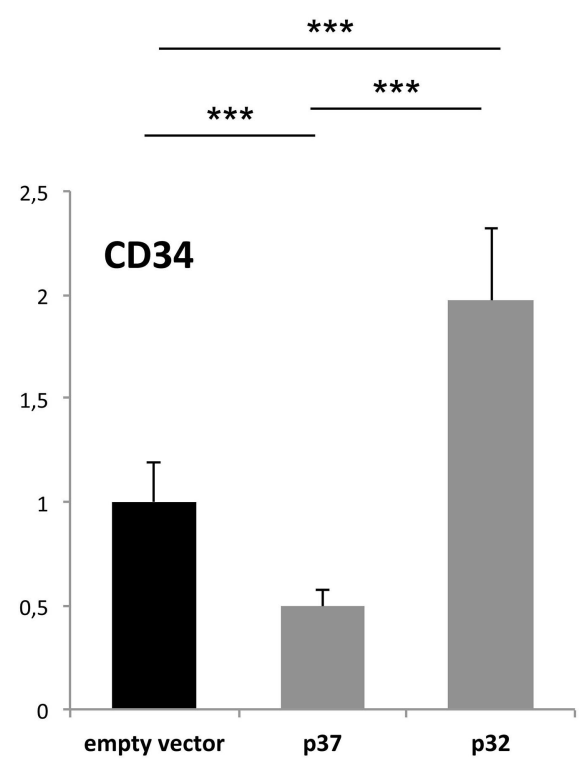

B

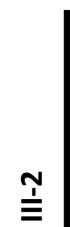

CD41

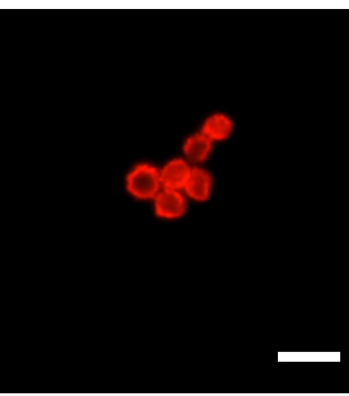

\section{CD34}

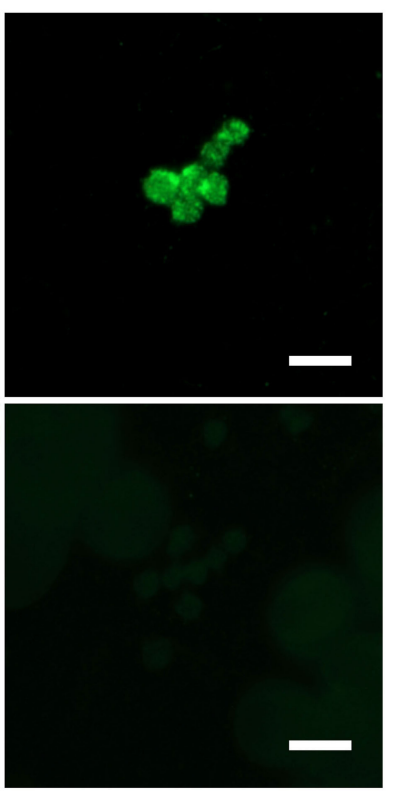

Figure 4. CD34 expression in c.648+5G>A patients. (A) Transcriptional activity of p37 (wild-type) and the variant p32 GFI1B isoforms measured on the CD34 promoter after transient transfection in Meg01 cells. Results are represented by the ratio of the promoter signal (Firefly) and the control promoter (Renilla) and expressed as fold change in luciferase activity. Significative variations occur on CD34 transcriptional levels as a significant p32 impaired repression activity is detected in comparison to p37 active repression ( $* * * P<0.001)$. Error bars represent the standard deviation of three independent experiments. Statistical analysis was performed using $t$-test. (B) Peripheral blood slides of the proband III-2 and healthy volunteers were double-labeled for CD41 (red) and CD34 (green). Platelets were identified by morphology and CD41 expression. Platelets of the proband III-2 showed a clear aberrant expression of the CD34 antigen compared to healthy control (HC). Scale bars correspond to $5 \mu \mathrm{m}$. 
could be responsible for thrombocytopenia. In fact, considering that p37 has a pivotal role in megakaryopoiesis and platelet production and that p32 is essential for erythroid lineage, distortion of the p32/p37 ratio is likely to dysregulate hemopoiesis. On the contrary, the role of p39 and p34, which represent approximately $3 \%$ and $10 \%$, respectively of the total GFIIB mRNA in both specimens of control samples, has not yet been defined?

We further investigated the role of p37 and p32 in terms of cellular localization and ability to control the transcription of targeted genes. In overexpressed conditions, most of the two isoforms are in the nucleus at comparable level, though their transcriptional effect is opposite. Whereas p37 represses the activity of the luciferase that is under the control of GFI1B target promoters, such as MEIS1, GFI1, and GFI1B itself, p32 increased its activity, reversing the transcriptional repression.

The same effect of c. $648+5 \mathrm{G}>\mathrm{A}$ on alternative splicing has previously been reported for c.648+1_648+8del GTGGGCAC, another mutation of GFI1B that was found in members of one family affected with MYH9related disease. ${ }^{10}$ As in our family, in individuals carrying the GFI1B but not the MYH9 variant, the expression level of p32 was markedly increased, being in at least equal amounts to p37. However, these individuals were not thrombocytopenic (mean value of $228 \times 10^{9} / \mathrm{L}$ ).

Another GFI1B variant, associated with skipping of exon 9 and significant reduction of the p37 expression level, is the synonymous substitution c.576C $>\mathrm{T}$ (p.Phe192=) in exon 9, which is reported as rs 150813342 with a MAF of $0.0041{ }^{28}$ Indeed, in individuals heterozygous for c.576C>T the platelet count is reduced with an average of 25$30 \times 10^{9} / \mathrm{L}$ in comparison with controls.

Finally, unbalance between p32 and p37 was identified in association with another mutation (c.551insG/p.Ser185Leufs*3). ${ }^{29}$ In patients homozygous for this mutation, while the expression level of p37 was significantly decreased, likely due to degradation by nonsense-mediated decay, the expression of p32 was the same level as healthy controls. Therefore, in these patients the active form of GFI1B is mainly p32. Of note, whereas individuals homozygous for c.551insG had a severe phenotype, heterozygous individulas were clinically unaffected. Of note, in these patients, as well as in our affected individuals, erythropoiesis was not defective, except for mild anisocytosis, suggesting that red cell production is rather independent of the p32/p37 ratio. ${ }^{29}$

Taken together all these observations suggested that the expressivity is extremely variable even when the variants exert the same effect. Indeed, bleeding and platelet count vary significantly, from individuals without defects (c.648+1_648+8delGTGGGCAC) or slight reduction of platelet count (c.576C>T) to those with an intermediate phenotype characterized by mild thrombocytopenia with mild bleeding tendency as in our family $(\mathrm{c} .648+5 \mathrm{G}>\mathrm{A})$ or with severe disease when the c.551ins mutation is homozygous ${ }^{29}$ Of note, abnormalities in megakaryocyte maturation were observed in isogenic cell clones homozygous for rs150813342 and in hematopoietic stem cell progenitors in which GFI1B p37 was selectively silenced ${ }^{28}$ suggesting that both expressivity and penetrance of the platelet phenotype might depend - at least in the cases enlisted above - on a specific threshold of the p32/p37 ratio, a value that cannot be estimated from the literature or our own data.

One feature that is likely to be pathognomonic of the GFI1B-associated bleeding disorder is the abnormal expression of CD34 on the platelet surface. ${ }^{10}$ Indeed, platelets of affected individual III-2, as well as those mentioned above with an altered p32/p37 ratio, express the CD34 antigen. Strong expression has also been reported in association with the Cys168Phe, Arg184Pro, and His181Tyr mutations, all affecting ZNF 1, or H294fsX307, Gln287* and G272fsX2 removing ZNF 5 and 6.,10-12

Changes in proportion between $\mathrm{p} 37$ and p32 significantly increasing p32 levels has been reported in acute and chronic leukemia, which could be interpreted as a triggering event for carcinogenesis. ${ }^{26}$ This hypothesis is supported by the

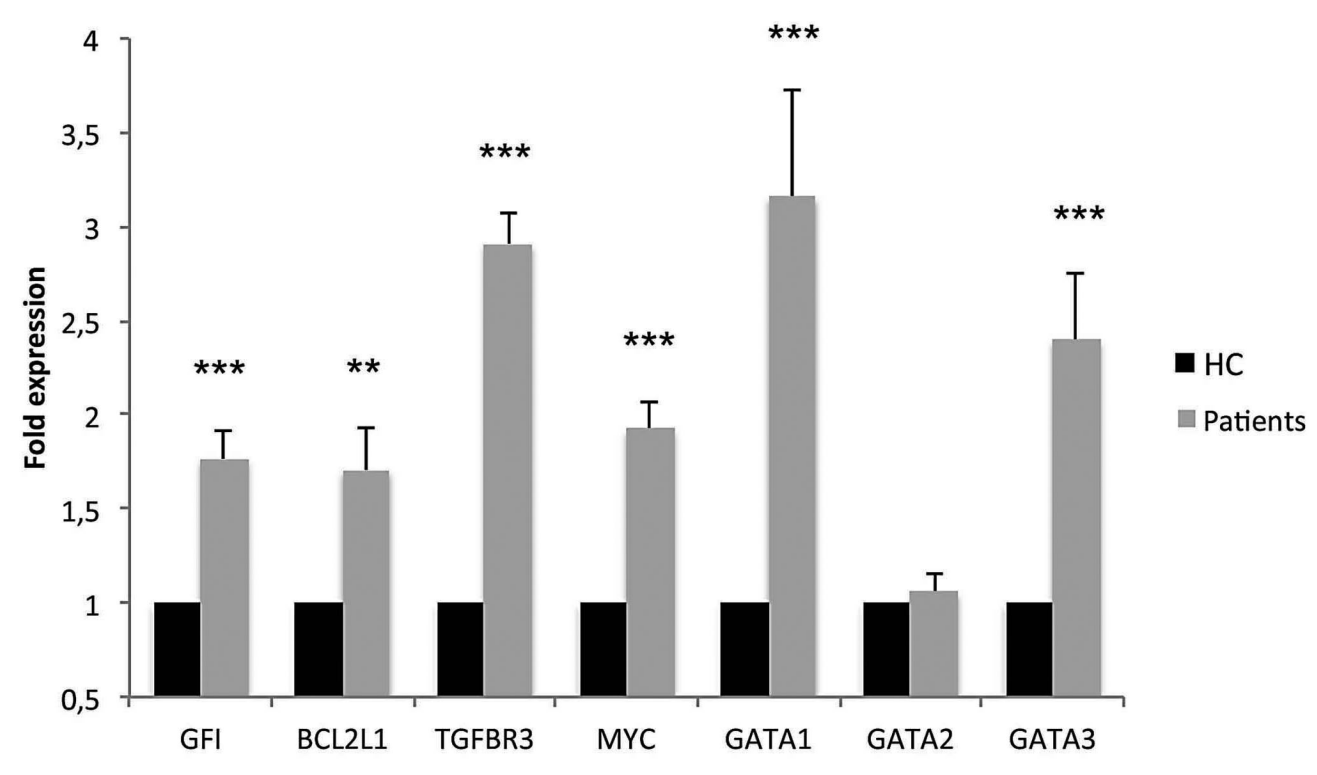

Figure 5. Quantitative polymerase chain reaction of GFI1B transcriptional target genes. The GFI, BCL2L1, TGF $\beta R 3$, MYC, GATA1, and GATA3 mRNA expression levels were significantly increased in patients (II-2 and III-1) compared to controls (two unrelated healthy individuals [HC]) (fold change from 1.5 to $>3$ ). No significant difference was observed for GATA2. Error bars represent the standard deviation of three independent experiments. Statistical analysis was performed using $t$-test. 
significant upregulation of GFI1, BCL2L1, TGFBR3, MYC, GATA1, and GATA3 in cells from the patients' peripheral blood. These genes are also differentially expressed in HEK cells overexpressing p32 or p37, confirming that p32 controls their transcription. Considering that these oncogenes are involved in hematopoietic differentiation and are dysregulated in various types of cancer, including hematological malignancies ${ }^{27}$ it would be interesting to establish whether the GFI1B-associated bleeding disorder could be part of the nosological entity called "Inherited thrombocytopenia-associated genes with predisposition to neoplasms". ${ }^{30}$

Increasing the detected number of individuals with $G F I 1 B$ mutations and creating a registry of individuals with $G F I 1 B$ variants, including a comprehensive medical and family history and regular follow-up, would be of fundamental importance in defining whether there is any increased risk of developing such hematological malignancies. Therefore, an effort should be made to identify patients with this rare form of thrombocytopenia by evaluating the CD34 expression on platelets, a useful assay in defining the pathogenicity of variants that would otherwise be considered of uncertain significance.

\section{Disclosures}

No conflicts of interest to disclose.

\section{Contributions}

$M F, N P, M C M K$, and RB performed research; $C M, T G$, and MS performed data analysis; FM, $P N$ and AP collected clinical data; $M F, S A$, and RB designed the study and wrote the manuscript; VB performed blood cell studies. All contributors have read and approved the manuscript.

\section{Acknowledgement}

The authors would like to thank patients for their participation in this project and Prof. Gughi D.L. for the constructive discussions.

\section{Funding}

This study was supported by IRCCS "Burlo Garofolo" (Ricerca Corrente 01/2018) and AIRC Grant IG-21974.

\section{References}

1. Noris P, Pecci A. Hereditary thrombocytopenias: a growing list of disorders. Hematology Am Soc Hematol Educ Program. 2017;2017(1):385-399.

2. Stevenson WS, Morel-Kopp MC, Chen Q, et al. GFI1B mutation causes a bleeding disorder with abnormal platelet function. I Thromb Haemost. 2013;11(11):2039-2047.

3. Monteferrario D, Bolar NA, Marneth AE, et al. A dominant-negative GFI1B mutation in the gray platelet syndrome. $\mathrm{N}$ Engl J Med. 2014;370(3):245-253.

4. Rabbolini DJ, Morel-Kopp MC, Ward CM, Stevenson WS. GFI1B variants associated with thrombocytopenia. Platelets. 2017;28 (5):525-527.

5. Zweidler-Mckay PA, Grimes HL, Flubacher MM, Tsichlis PN. Gfi-1 encodes a nuclear zinc finger protein that binds DNA and functions as a transcriptional repressor. Mol Cell Biol. 1996;16(8):4024-4034.

6. Vassen L, Fiolka K, Mahlmann S, Möröy T. Direct transcriptional repression of the genes encoding the zinc-finger proteins Gfilb and Gfil by Gfilb. Nucleic Acids Res. 2005;33(3):987-998.

7. Laurent B, Randrianarison-Huetz V, Frisan E, et al. A short Gfi-1B isoform controls erythroid differentiation by recruiting the LSD1-CoREST complex through the dimethylation of its SNAG domain. J Cell Sci. 2012;125(Pt 4):993-1002.

8. Khandanpour C, Sharif-Askari E, Vassen L, et al. Evidence that growth factor independence $1 \mathrm{~b}$ regulates dormancy and peripheral blood mobilization of hematopoietic stem cells. Blood. 2010;116(24):5149-5161.

9. Randrianarison-Huetz V, Laurent B, Bardet V, Blobe GC, Huetz F, Duménil D. Gfi-1B controls human erythroid and megakaryocytic differentiation by regulating TGFbeta signaling at the bipotent erythromegakaryocytic progenitor stage. Blood. 2010;115(14):2784-2795

10. Rabbolini DJ, Morel-Kopp MC, Chen Q, et al. Thrombocytopenia and CD34 expression is decoupled from $\alpha$-granule deficiency with mutation of the first growth factor-independent $1 \mathrm{~B}$ zinc finger. J Thromb Haemost. 2017; 15(11):2245-2258.

11. van Oorschot $R$, Marneth AE, Bergevoet $\mathrm{SM}$, et al. Inherited missense variants that affect GFI1B function do not necessarily cause bleeding diatheses. Haematologica. 2019;104(6):e260-e4

12. Kitamura K, Okuno Y, Yoshida K, et al. Functional characterization of a novel GFI1B mutation causing congenital macrothrombocytopenia. J Thromb Haemost. 2016;14 (7):1462-1469.

13. Ferreira CR, Chen D, Abraham SM, et al Combined alpha-delta platelet storage pool deficiency is associated with mutations in GFI1B. Mol Genet Metab. 2017;120(3):288294.

14. Uchiyama Y, Ogawa Y, Kunishima S, et al. A novel GFI1B mutation at the first zinc finger domain causes congenital macrothrombocytopenia. Br J Haematol. 2018;181(6):843847.

15. Chen L, Kostadima M, Martens JHA, et al. Transcriptional diversity during lineage commitment of human blood progenitors. Science. 2014;345(6204):1251033.

16. Cheng AN, Bao EL, Gughi DL, Fiorini C, Sankaran VG. Macrothrombocytopenia associated with a rare GFI1B missense variant confounding the presentation of immune thrombocytopenia. Pediatr Blood Cancer. 2019;66(9):e27874.

17. Johnson B, Lowe GC, Futterer J, et al. Whole exome sequencing identifies genetic variants in inherited thrombocytopenia with secondary qualitative function defects. Haematologica. 2016;101(10):1170-1179.

18. Noris P, Biino G, Pecci A, et al. Platelet diameters in inherited thrombocytopenias: analysis of 376 patients with all known disorders. Blood. 2014;124(6):e4-e10.

19. Noris P, Guidetti GF, Conti V, et al. Autosomal dominant thrombocytopenias with reduced expression of glycoprotein Ia Thromb Haemost. 2006;95(3):483-489.

20. Marconi C, Di Buduo CA, Barozzi S, et al. SLFN14-related thrombocytopenia: identification within a large series of patients with inherited thrombocytopenia. Thromb
Haemost. 2016;115(5):1076-1079.

21. Bottega R, Pecci A, De Candia E, et al Correlation between platelet phenotype and NBEAL2 genotype in patients with congenital thrombocytopenia and $\alpha$-granule deficiency. Haematologica. 2013;98(6):868-874.

22. De Rocco D, Melazzini F, Marconi C, et al. Mutations of RUNX1 in families with inherited thrombocytopenia. Am J Hematol 2017;92(6):E86-E8.

23. Bottega R, Nicchia E, Cappelli E, et al Hypomorphic FANCA mutations correlate with mild mitochondrial and clinical phenotype in Fanconi anemia. Haematologica. 2018;103(3):417-426.

24. Faleschini M, Melazzini F, Marconi $C$, et al. ACTN1 mutations lead to a benign form of platelet macrocytosis not always associated with thrombocytopenia. Br J Haematol. 2018;183(2):276-288.

25. Balduini CL, Pecci A, Loffredo G, et al Effects of the R2160 mutation of GATA-1 on erythropoiesis and megakaryocytopoiesis. Thromb Haemost. 2004;91(1) 129-140.

26. Vassen L, Khandanpour C, Ebeling P, et al. Growth factor independent $1 \mathrm{~b}$ (Gfilb) and a new splice variant of Gfilb are highly expressed in patients with acute and chronic leukemia. Int J Hematol. 2009;89(4):422-430

27. Anguita E, Candel FI, Chaparro A, Benet M, Roldán-Etcheverry JJ. Transcription factor GFI1B in health and disease. Front Oncol 2017;7:54.

28. Polfus LM, Khajuria RK, Schick UM, et al Whole-exome sequencing identifies loci associated with blood cell traits and reveals a role for alternative GFI1B splice variants in human hematopoiesis. Am J Hum Genet. 2016;99(3):785

29. Schulze H, Schlagenhauf A, Manukjan G, et al. Recessive grey platelet-like syndrome with unaffected erythropoiesis in the absence of the splice isoform GFI1B-p37. Haematologica. 2017;102(9):e375-e378.

30. Arber DA, Orazi A, Hasserijian R, et al. The 2016 revision to the World Health Organization classification of myeloid neoplasms and acute leukemia. Blood 2016;127(20):2391-2405. 